\title{
Simple Vector Considerations to Assess the Polarity of Partially Fluorinated Alkyl and Alkoxy Groups
}

\author{
Klaus Müller*
}

\begin{abstract}
While $\mathrm{H} / \mathrm{F}$ exchange at aryl groups or introduction of $\mathrm{CF}_{3}$ units have dominated medicinal chemistry for decades, the use of partially fluorinated alkyl and alkoxy groups has come into focus more recently. A simple bond vector analysis scheme, based on the assumption of context-independent bond polarities as well as idealized configurational and conformational geometries, is applied to small alkyl and alkoxy groups with prototypic fluorination patterns for qualitative assessments of relative polarities as well as polarity modulation by conformational change. Combined with a constant volume increase for each hydrogen/fluorine exchange these polarity estimates can be translated into expected lipophilicity shifts $(\Delta \log P)$, using a simple parameterization scheme derived from experimental $\log P$ values. While terminal monofluoro- and gem-difluoromethyl groups in small aliphatic units are correctly predicted to show lower lipophilicity compared to their tri- or non-fluorinated congeners, vicinal difluoro and bis-vicinal trifluoro patterns are identified to exhibit significantly lower lipophilicities than their respective geminal di- and trifluoro substituted counterparts. The trifluoromethoxy group is diagnosed as an intrinsically lipophilic unit compared to the parent methoxy group. The difluoromethoxy group is of particular interest as it can easily interconvert between a highly lipophilic and a polar conformation, thus enabling this unit to adjust to polarity changes of the molecular environment. For di- and trifluoroalkoxy groups, again the vic-difluoro and bis-vic-trifluoro substitution patterns stand out as most promising to keep lipophilicity low. The 1,3-difluoro pattern next to an ether moiety shows an interesting conformational dependence of polarity, similar to the difluoromethoxy group. While very qualitative in nature, the simple bond vector analysis promises to be a useful tool for the identification of lipophilicity-lowering fluorinated alkyl or alkoxy groups and particularly those groups that display marked conformation-dependent lipophilicity and thus potentially serve as 'environmental adaptors'.
\end{abstract}

Keywords: Fluorinated alkoxy groups · Fluorinated alkyl groups · Lipophilicity · Polarity · Vector analysis

Fluoroorganic chemistry keeps fascinating all branches of the Life and Material Sciences. Fluorine's special position in the periodic table with its extreme electronegativity and strict monovalent binding mode, its small size and little polarizability when covalently bound render hydrogen-fluorine exchange a rewarding concept for distinct modulation of compound properties. While introduction of fluorine into organic compounds has been described for more than 100 years, ${ }^{[1]}$ and the first fluorinecontaining drug was introduced almost 60 years ago, ${ }^{[2]}$ a more systematic assessment of the many changes in compound properties due to fluorine-hydrogen exchange appeared only some two decades ago with the seminal review by Bruce Smart. ${ }^{[3]}$ Dramatic improvements of synthetic methodology and increasing availability

\footnotetext{
${ }^{\star}$ Correspondence: Prof. K. Müller

F. Hoffmann-La Roche AG

Pharma Research \& Early Development

$\mathrm{CH}-4070$ Basel, Switzerland

Tel.: +41616884075

E-mail: klaus.mueller@roche.com
}

of F-containing synthetic building blocks have expanded the field enormously over the recent years with concomitant enrichment of property data of fluorine-containing compounds. International symposia and fluorine workshops at regular intervals on fluoroorganic chemistry followed by special issues of journals summarizing the highlights of such events, ${ }^{[4]}$ as well as a series of reviews ${ }^{[5]}$ document the increasing understanding of the specific effects that fluorine exerts on compounds to which it is grafted. The most prominent effects of hydrogen-fluorine exchange on compound properties are schematically summarized in Fig. 1.

The highly polarized carbon-fluorine bond not only results in a marked local polarity, but also polarizes the molecule at considerable distances from the $\mathrm{C}-\mathrm{F}$ bond. This is particularly evident from the $p \mathrm{~K}_{\mathrm{a}}$ values of amine functions in the environment of a C-F bond. Thus, stepwise introduction of a fluorine atom in $\beta$-position to an amine lowers the $p \mathrm{~K}_{\mathrm{a}}$ of the amino group systematically by about $1.7 p \mathrm{~K}_{\mathrm{a}}$ units per fluorine atom. ${ }^{[5 \mathrm{~b}, 6]}$ On the other hand, removal of the site of fluorination from the amino function results in an exponential attenuation of the $p \mathrm{~K}_{\mathrm{a}}$ lower- ing effect as a function of topological distance. Since a $\mathrm{CF}_{3}$ group in $\alpha$-position to an amino group (equivalent to three fluorine atoms in $\beta$-position), has a dramatic $p \mathrm{~K}_{\mathrm{a}}$-lowering effect, a $\mathrm{CF}_{3}$ group in a remote $\delta$-position can still exert a small but distinct $p \mathrm{~K}_{\mathrm{a}}$ depression. Likewise, since such $p \mathrm{~K}_{\mathrm{a}}$-modulating effects are largely additive in conformationally unrestricted acyclic systems, small but distinct $p \mathrm{~K}_{\mathrm{a}}$ depressions can be expected even for quite remote single fluorine atoms. Such effects can be used to optimize potential drug candidates. A strong conformational dependence of the $p \mathrm{~K}_{\mathrm{a}}$ depression is well documented for piperidine derivatives, where fluorine atoms in the axial position typically exert a much smaller basicity lowering effect than a fluorine substituent in the corresponding equatorial position. This is true for fluorine substitutions in both $\beta$ - and $\gamma$-positions. A preferred transmission of polarization through a contiguous antiperiplanar bond network may account for these observations. For an axial fluorine atom in $\beta$-position, a stabilizing antiperiplanar $\mathrm{H}-\mathrm{N} \cdots \mathrm{C}-\mathrm{F}$ dipole-dipole interaction may significantly contribute to this effect (see below). Substituent effects on piperidine basicity are of particular 


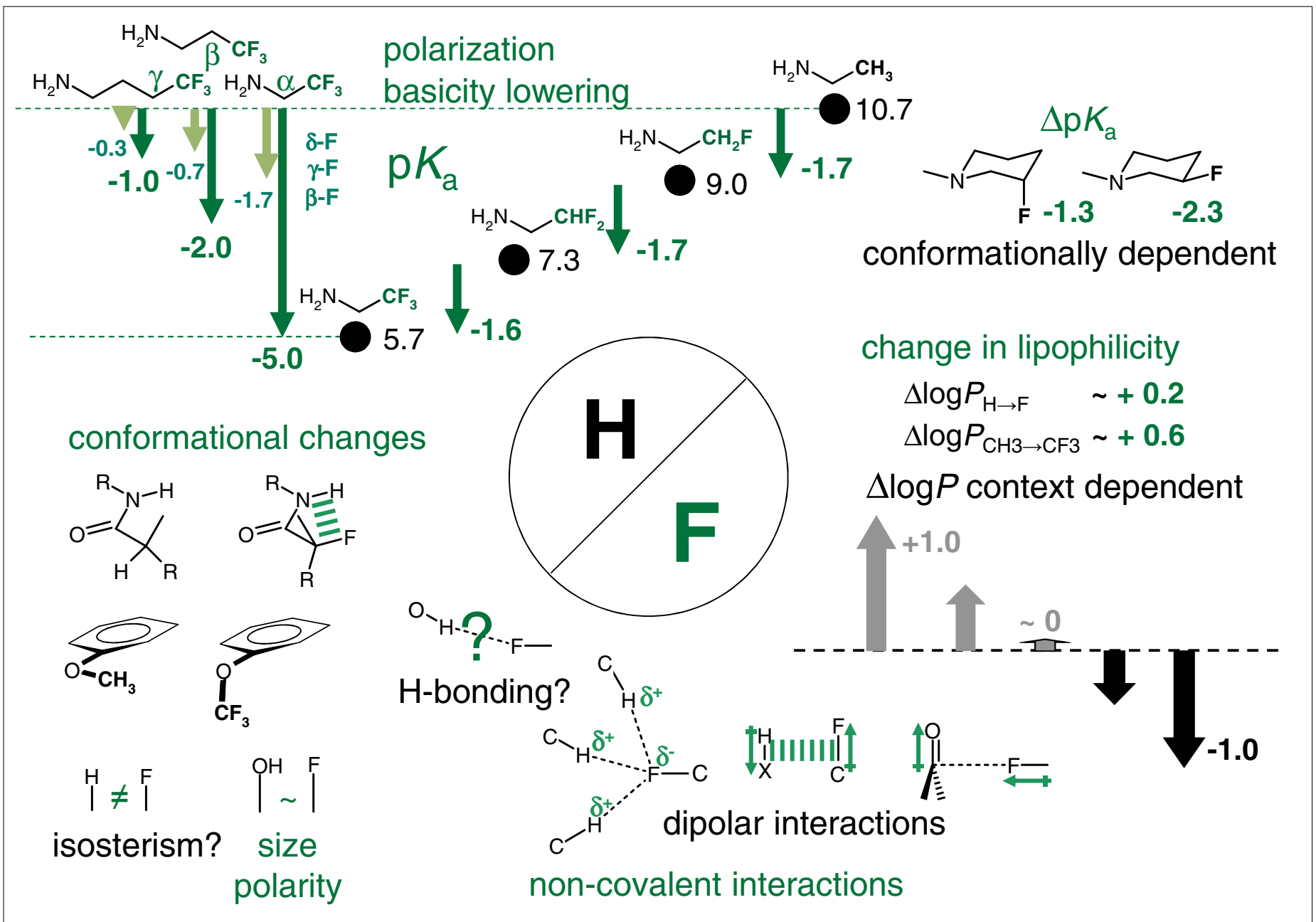

Fig. 1. Summary of effects on compound properties due to hydrogen-fluorine exchange.

interest, as piperidine with its chair-type well-staggered conformation may serve as reference for substituted acyclic, but conformationally constrained amines.

Hydrogen-fluorine exchange is often accompanied by a slight but distinct lipophilicity increase. Thus, exchange of an aromatic $\mathrm{C}-\mathrm{H}$ by the $\mathrm{C}-\mathrm{F}$ bond results typically in a slight increase of $0.1-03$ $\log P$ units. Likewise, replacement of an aromatic methyl group by a trifluoromethyl group may produce $\log P$ upshifts of 0.6 units. However, these $\log P$ changes appear to depend on the overall lipophilicity of the parent (neutral) compound. ${ }^{[7]}$ With increasing lipophilicity of the compound the local polarity of the $\mathrm{CF}_{3}$ group may dominate over the concomitant $\mathrm{CH}_{3} / \mathrm{CF}_{3}$ volume increase and thus significantly reduce the $\log \mathrm{P}$ gain.

It has been noted already quite early ${ }^{[3 b]}$ that the largely positive $\log P$ changes observed for $\mathrm{H} / \mathrm{F}$ exchanges at aromatic cores contrast the often negative $\Delta \log P$ effects when either a single hydrogen or a methyl group in aliphatic systems are replaced by a fluorine atom or $\mathrm{CF}_{3}$ group, respectively. In a more systematic study of a series of alkyl-substituted indole derivatives it could be shown that both the monofluoro and difluoromethyl groups exhibit stronger polarity, hence lower lipophilicities, than either the parent methyl group or its trifluoromethyl counterpart. [7] These effects could be rationalized in terms of the partial compensation of total local polarity effects and small $\mathrm{H} / \mathrm{F}$ volume increases. Overall, $\log P$ variations upon $\mathrm{H} / \mathrm{F}$-exchange turn out to vary substantially according to the local structural contexts, ranging from $\Delta \log P \sim+1$ or even higher to $\Delta \log P \sim-1$ (Fig. 1). Furthermore, changes in $\log P$ also depend on characteristic fluorination patterns (see below). A structure-based understanding of these effects provides powerful tools in the design and optimization of drug candidates.

After quite some debate regarding the hydrogen bond acceptor capacity of covalently bound fluorine and in spite of sporadically recurrent claims of $\mathrm{X}-\mathrm{H} \cdots \mathrm{F}-\mathrm{C}$ hydrogen bonding in the literature, ${ }^{[8]}$ there is convincing evidence both in solution ${ }^{[9]}$ and the solid state ${ }^{[10]}$ that covalent fluorine hardly engages in hydrogen bonding. Significant non-covalent interactions of $\mathrm{C}-\mathrm{F}$ units are probably mostly of a weak electrostatic di- or multipolar nature, ${ }^{[11]}$ as summarized in Fig. 1. The most frequent mode of intermolecular interactions by a
$\mathrm{C}-\mathrm{F}$ unit is found in both protein-molecular complexes and in the crystal packing of fluorine-containing compounds, where $\mathrm{C}-\mathrm{F}$ units are bound in lipophilic pockets with weakly positively polarized $\mathrm{C}-\mathrm{H}$ bonds as exemplified, e.g., by the wellresolved crystal structure of the ternary complex of an allosteric inhibitor (GNF-2) and the ATP-competitive inhibitor imatinib (Gleevec) to the Bcr-Abl kinase (Fig. 2). ${ }^{[12]}$

A particularly instructive example of crystal packing of a small F-containing molecule is given by 1,6-anhydro-2,4deoxy-2,4-difluoro- $\beta$-D-glucopyranose (Fig. 3):[13] This difluoro anhydrosugar contains one $\mathrm{OH}$ group that can interact either with $\mathrm{OH}$ groups, ether oxygen or well-exposed fluorine atoms of neighboring molecules. There is a continuous zigzag chain of hydroxyl groups with alternating distances of $d_{O} \ldots=3.25 \AA$ and $3.64 \AA$. These inter-oxygen distances are too large to sustain a chain of cooperative hydrogen bonds. Indeed, the $\mathrm{OH}$ groups of the two independent molecules in the asymmetric unit predominantly interact via hydrogen bonds to 1',6'-ether oxygen atoms of neighboring molecules at distances of $d_{0} \ldots$ $=2.80 \AA$ and $2.97 \AA$, respectively. The $\mathrm{OH}$ groups do not care about the fluorine li- 


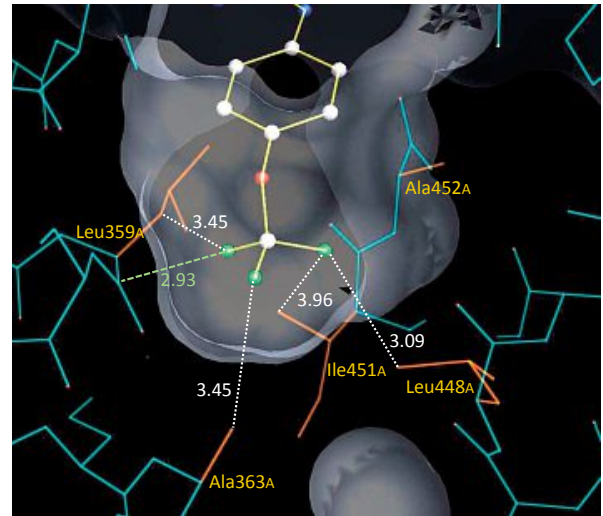

Fig. 2. Binding of the $\mathrm{CF}_{3}$ group of the trifluoromethoxyphenyl unit of the non-ATP-competitive inhibitor GNF-2 in the hydrophobic pocket of Bcr-Abl kinase (PDB code: 3K5V; A-chain); the close contacts of the three $F$ atoms to hydrophobic residues are indicated with dotted white lines (distances in Å); note that one $F$ atom also engages in an orthogonal dipolar interaction with the peptide carbonyl group of Leu359 (numbering scheme for amino acids are those given in the deposited PDB file; they are shifted by +19 with respect to those used in ref. [12]).

gands. The exposed fluorine atoms on the other hand are exclusively in close contacts with polarized $\mathrm{C}-\mathrm{H}$ bonds. Note that in this difluorinated anhydrosugar derivative, all $\mathrm{C}-\mathrm{H}$ bonds are flanked by at least one $\mathrm{C}-\mathrm{O}$ or $\mathrm{C}-\mathrm{F}$ bond, which increases their polarity and thus partial positive charges at the hydrogen atoms. Each fluorine atom in the two independent molecules interacts with four close $\mathrm{C}-\mathrm{H}$ bonds of neighboring molecules with distances $\mathrm{d}_{\mathrm{F}} \cdots_{\mathrm{HC}} \leq 3.00 \AA$. These distances scatter around $2.74 \AA$ $\pm 0.19 \AA$, thus close to the $\mathrm{F} \cdots \mathrm{H}$ van $\mathrm{der}$ Waals contact distance $(2.67 \AA)$ between covalent fluorine and hydrogen atoms.

A second important non-covalent interaction mode of the $\mathrm{C}-\mathrm{F}$ unit is the $(1,3)$-antiparallel dipolar interaction with a polar $\mathrm{X}-\mathrm{Y}$ bond unit. This interaction mode occurs mainly intramolecularly and accounts for the stabilization of protonated cyclic amines with (pseudo)axial $\mathrm{C}-\mathrm{F}$ units in $\beta$-position (see Fig. 1, top right corner), the partial shielding of polar $\mathrm{OH}$ groups in $\beta$-fluorinated alcohols ${ }^{[14]}$ with markedly increased lipophilicities (going from ethanol to 2,2,2-trifluoro-ethanol results in a lipophilicity upshift of $\Delta \log P \sim+0.7,{ }^{[3 b]}$ in spite of the increased acidity of trifluoroethanol, whereas introduction of three terminal fluorines in the homologous $n$-propanol results in only a marginal $\log P$ upshift of +0.1 or no upshift at all in the case of $n$-butanol[3b]), as well as in conformational preferences in specific cases where $\mathrm{OH}$ or $\mathrm{NH}$ groups can align essentially in plane with a $\beta$-positioned $\mathrm{C}-\mathrm{F}$ unit. ${ }^{[5 \mathrm{~d}, 15]}$ However, weak but favorable dipolar interaction modes can also be expected to operate between the less polarized $\mathrm{C}-\mathrm{H}$ units in
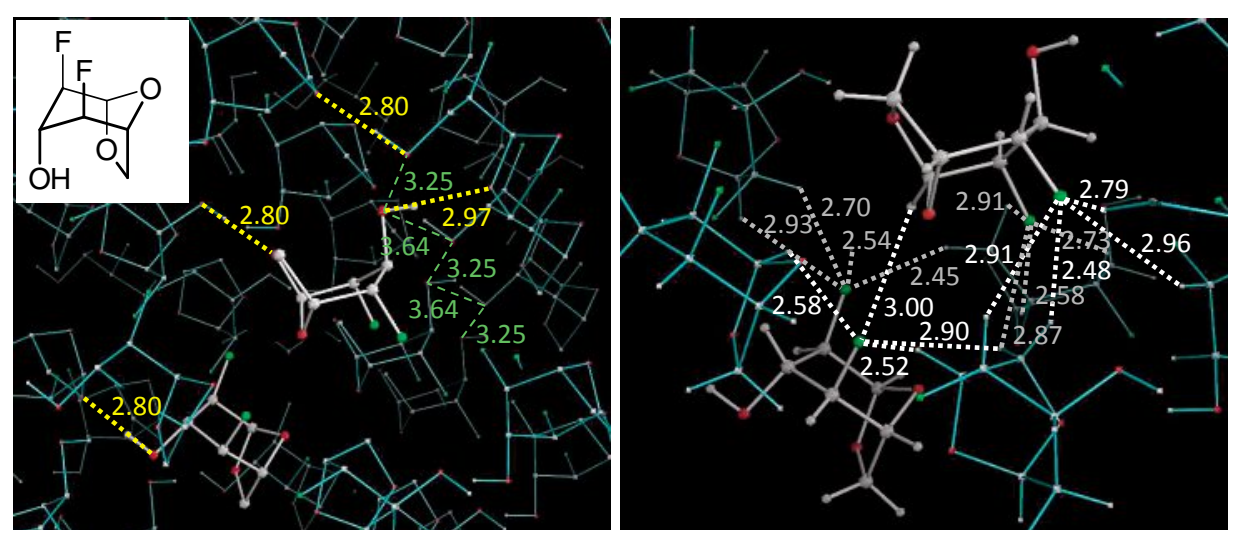

Fig. 3. Molecular structure and crystal packing of 1,6-anhydro-2,4-deoxy-2,4-difluoro- $\beta$-Dglucopyranose $^{[13]}$ as retrieved from Cambridge Structural Database (CSD code ADFGLP). proper arrangement relative to a $\mathrm{C}-\mathrm{F}$ bond (Fig. 1).

Finally, a C-F unit has been frequently found to interact in an orthogonal dipolar interaction mode with electrophilic systems such as the $\mathrm{C}=\mathrm{O}$ bond of carbonyl derivatives. ${ }^{[16]}$ This interaction mode may operate both intra- and intermolecularly and is of course not limited to $\mathrm{C}-\mathrm{F} \cdots \mathrm{C}=\mathrm{O}$ interactions, but can be encountered with electrophilic system $\mathrm{E}=\mathrm{W}$.

The absolute magnitude of these types of C-F dipolar interactions is small, ranging typically from $0.1-1.0 \mathrm{kcal} / \mathrm{mole}$, the lower range applying particularly to $\mathrm{C}-\mathrm{F} *$ $\mathrm{H}-\mathrm{C}$ interactions, whereas (1,3)-antiparallel or orthogonal interaction modes may result in more favorable energy gains. ${ }^{[16]}$ However, since the interaction of a $\mathrm{C}-\mathrm{F}$ unit in a hydrophobic pocket typically involves numerous $\mathrm{C}-\mathrm{F} \cdots \mathrm{H}-\mathrm{C}$ contacts, small but significant energy contributions can be expected to result.

The size of a $\mathrm{C}-\mathrm{F}$ unit versus $\mathrm{C}-\mathrm{H}$ and in particular that of a $\mathrm{CF}_{3}$ versus $\mathrm{CH}_{3}$ group have been of some controversy in the past. $[3 b, 11,17,18]$ any polar unit $\mathrm{X}-\mathrm{Y}$ interacting with an
Based on bond lengths and van der Waals radii the $\mathrm{C}-\mathrm{F}$ moiety exhibits a distinctly larger extension than a $\mathrm{C}-\mathrm{H}$ unit. Accordingly, the $\mathrm{C}-\mathrm{F}$ bond is considered to be more similar to a $\mathrm{C}-\mathrm{OH}$ unit, both in terms of polarity and dimension. ${ }^{[11]}$ Indeed, provided that the $\mathrm{H}$-bond donation capacity of the latter is taken care of by the local molecular environment, both units have been shown to exhibit strikingly similar modes of non-bonded interactions. ${ }^{[16]}$ In terms of van der Waals volumes, a single $\mathrm{H} / \mathrm{F}$ exchange results in a molecular volume increase of approximately $1 / 3$ of that calculated for an $\mathrm{H} / \mathrm{CH}_{3}$ exchange. Accordingly, the axially isotropic $\mathrm{CF}_{3}$ group occupies approximately double the volume of a $\mathrm{CH}_{3}$ group, consistent with bond lengths and van der Waals radii, and thus is smaller than the axially anisotropic isopropyl group. ${ }^{[18]}$

Size and polarity of $\mathrm{CF}$ units are important determinants of conformational effects. The aryl-bound trifluoromethoxy group constitutes a most prominent example. It generally adopts an orthogonal or nearly orthogonal arrangement to the aryl ring by contrast to parent methoxy
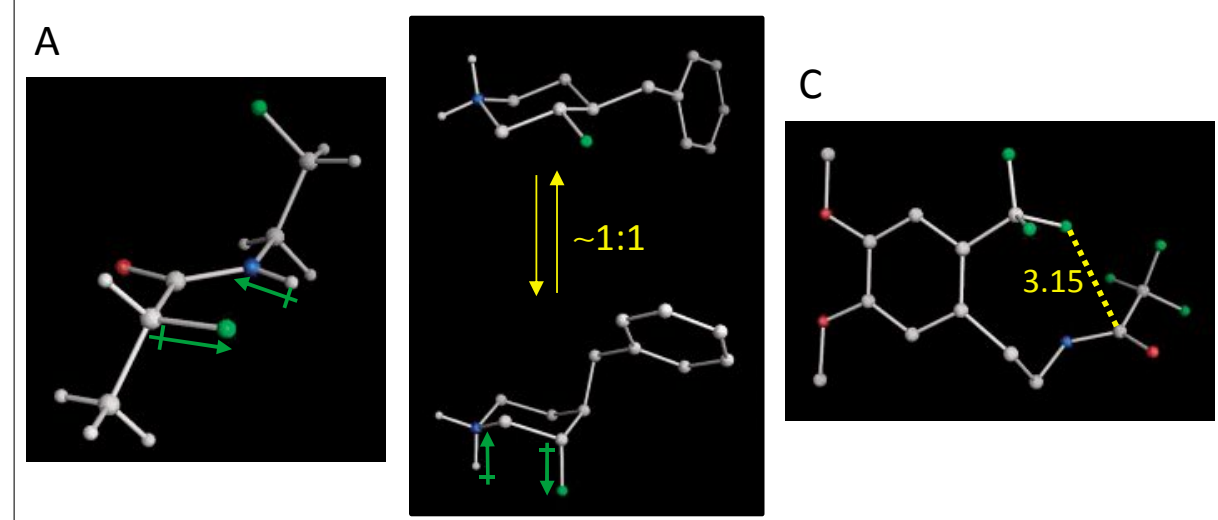

Fig. 4. Conformational arrangements favored by intramolecular (1,3)-antiparallel dipolar interactions between polar $\mathrm{C}-\mathrm{F}$ and $\mathrm{N}-\mathrm{H}$ bonds: (A) syn-planar arrangement of $\mathrm{C}-\mathrm{F}$ to $\mathrm{N}-\mathrm{H}$ in an $\alpha$-fluorocarboxamide; ${ }^{[15]}$ (B) (1:1) co-existence of diequatorial and diaxial conformations of protonated 3-fluoro-4-benzylpiperidine in $\mathrm{D}_{2} \mathrm{O} / \mathrm{DCl}$ by NMR; ${ }^{[5 b]}(\mathrm{C})$ preference of a compact gauche conformation in a dopamine derivative by a favorable intramolecular $\mathrm{C}-\mathrm{F} \cdots \mathrm{C}=\mathrm{O}$ orthogonal dipolar interaction (CSD-code $=$ YODVOC). ${ }^{[19]}$ 
derivatives ${ }^{[5 b]}$ which uniformly prefer essentially planar conformations when at least one ortho-position in the aryl moiety is unsubstituted. Favorable anomeric interactions between the oxygen lone pairs and antiperiplanar $\mathrm{C}-\mathrm{F}$ bonds of the $\mathrm{CF}_{3}$ group weaken the anisol-type $\pi$-conjugation, thus rendering the anisol moiety more sensitive to steric hindrance and forcing the bigger $\mathrm{CF}_{3}$ group into the orthogonal position. (1,3)-Antiparallel dipolar interactions between $\mathrm{C}-\mathrm{F}$ and polar $\mathrm{N}-\mathrm{H}$ or $\mathrm{O}-\mathrm{H}$ bonds result in distinct conformational arrangements, stabilizing a diaxial conformation in the $N$-protonated trans-3-fluoro-4-benzylpiperidine ${ }^{[6]}$ or favoring the in-plane arrangement of the $\mathrm{C}-\mathrm{F}$ bond with respect to the $\mathrm{N}-\mathrm{H}$ bond in $\alpha$-fluorinated amides. ${ }^{[5 \mathrm{~d}, 15]}$ Intramolecular orthogonal dipolar interactions between $\mathrm{C}-\mathrm{F}$ units and $\mathrm{C}=\mathrm{O}$ units may stabilize compact conformations, such as in $O, O^{\prime}$-dimethyl- $N$-trifluoroacetyl6-trifluoromethyldopamine exhibiting a double-gauche conformation in the phenethylamine moiety enabling a close contact $\left(\mathrm{d}_{\mathrm{F}} \cdots_{\mathrm{CO}}=3.15 \AA\right)$ between one fluorine atom of the $6-\mathrm{CF}_{3}$ group and the acetyl carbonyl C-atom (Fig. 4). [19]

Partially fluorinated methyl groups in aliphatic systems exert lower lipophilicities than the trifluoromethyl group as evidenced in $n$-propylbenzene and indole se$\operatorname{ries}^{[7]}$ (Fig. 5). These observations could be rationalized by simple polarity and volume considerations assuming proportionate downshifts of $\log P$ according to polarity changes upon $\mathrm{H} / \mathrm{F}$ exchange and counterbalancing $\log P$ upshifts by concomitant volume increases. [7] The simplest possible scheme, assuming context-independent C-F bond polarity vectors $\left(\mu_{\mathrm{CF}}\right)$ and tetrahedral configuration at saturated carbon atoms, provide a fair representation of the fact that difluoromethane is more polar than either the monofluoro- or trifluoromethane, correctly predicts monofluoro and difluoromethyl derivatives to have lower lipophilicity than the trifluoromethyl counterpart. However, examination of experimental gas-phase dipole moments of fluorinated ethane and propane derivatives reveals slight but systematic increases of corresponding dipole moments in going from methane to the higher alkane series, reflecting the enhanced possibilities for fluorine atoms to effect polarization through trans-arranged $\mathrm{C}-\mathrm{H}$ units or the alkane backbone. Furthermore, for a terminal $\mathrm{CF}_{3}$ group in the higher $n$-alkanes all three terminal fluorine atoms can effectively polarize through trans- $\mathrm{CH}$ units or the alkane backbone, whereas this is not possible in the parent trifluoromethane case. Hence, terminal trifluorination of the higher alkanes results in an increased, rather than decreased dipole moment, when compared to the monofluorinated

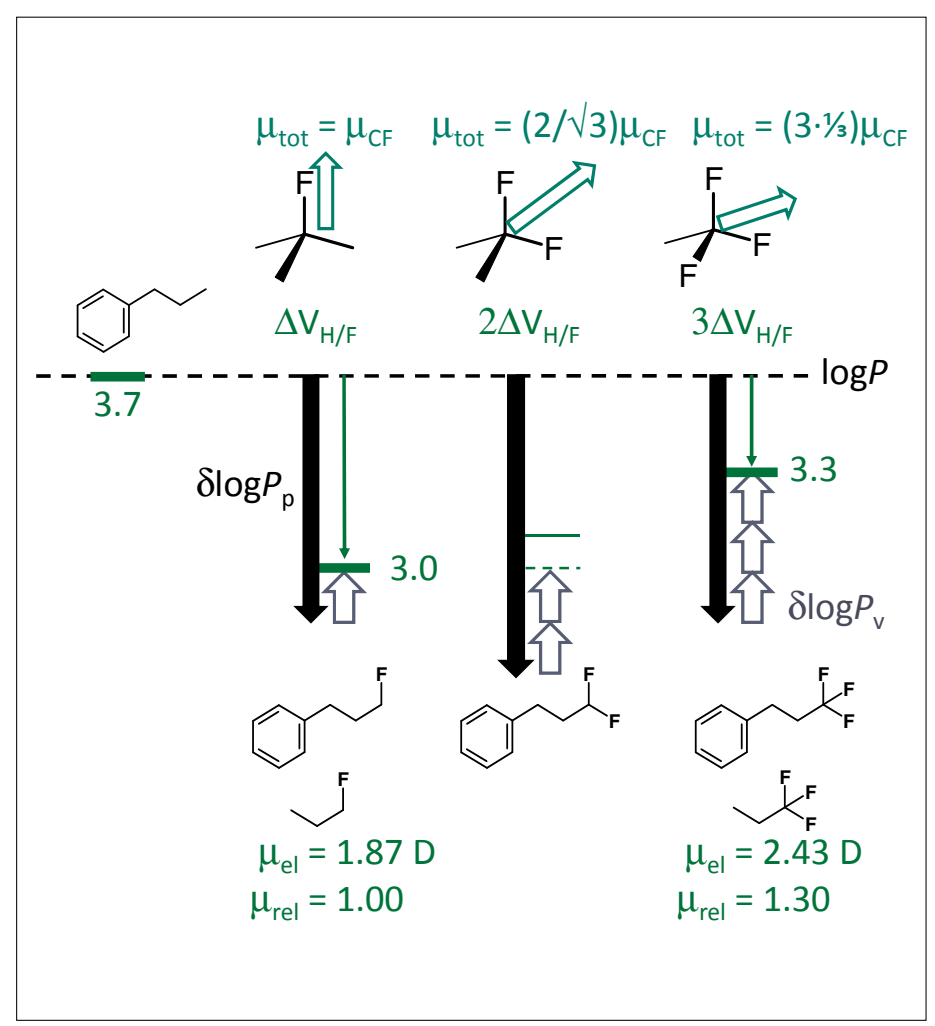

Fig. 5. Lipophilicities of terminally fluorinated $n$-propylbenzene derivatives and their rationalization based on a simple $\mathrm{C}-\mathrm{F}$ bond vector superposition model, refined according to experimental and theoretically estimated dipole moments of terminally fluorinated propane derivatives. Based on the experimental $\log P$ values, polarityinduced $\log P$ increments, $\Delta \log P_{\mathrm{p}} \sim-1.0$ per units of $\mu_{\mathrm{CF}}$, and volume-induced $\log P$ increments, $\delta \log P_{v}$ $\sim+0.3$ per single $\mathrm{H} / \mathrm{F}$ exchange are obtained. With these two parameters, expected $\Delta \log P$ shifts for different fluorination patterns can be roughly estimated.

congeners. Therefore, a revised $\mathrm{C}-\mathrm{F}$ bond vector model may be based directly on experimental and computed dipole moments of fluorinated propane derivatives, resulting in qualitatively similar $\log P$ predictions, except that the difluoromethyl unit is now predicted to be slightly less polar than the monofluoromethyl unit, but still more polar than its trifluoromethyl counterpart in accordance with experimental observations in different series. ${ }^{\text {[7] Gratifyingly, for }}$ all systems investigated, a gem-difluoro derivative exhibits small but distinct increases in polarity, consistent with an augmented dipole moment resulting from the superposition of two $\mathrm{C}-\mathrm{F}$ bond vectors in an essentially tetrahedral configuration.

Remarkably, vicinal difluoroalkane derivatives exhibit a markedly enhanced polarity compared to the corresponding gemdifluoro derivatives. This is well borne out by comparison of the experimental dipole moments of 1,2-difluoroethane in its favored gauche-conformation and 1,1-difluoroethane as well as by the theoretical calculations for 1,1- and 2,2-difluoropropane, as well as 1,2-difluoropropane in both the endo,endo-and exo,endo-conformations. ${ }^{[7]}$ The increase in polarity can be qualitatively traced to the different geometrical arrangements of the two $\mathrm{C}-\mathrm{F}$ bond vectors in gem- and vic-difluoro derivatives (Fig. 6).

Assuming perfectly staggered tetrahedral geometries for the gem- and vicdifluoroalkane model systems, and shifting the a vicinal $\mathrm{C}-\mathrm{F}$ bond vector into the origin of the other $\mathrm{C}-\mathrm{F}$ bond vector, it is immediately evident that geminal and vicinal difluorination patterns differ only,

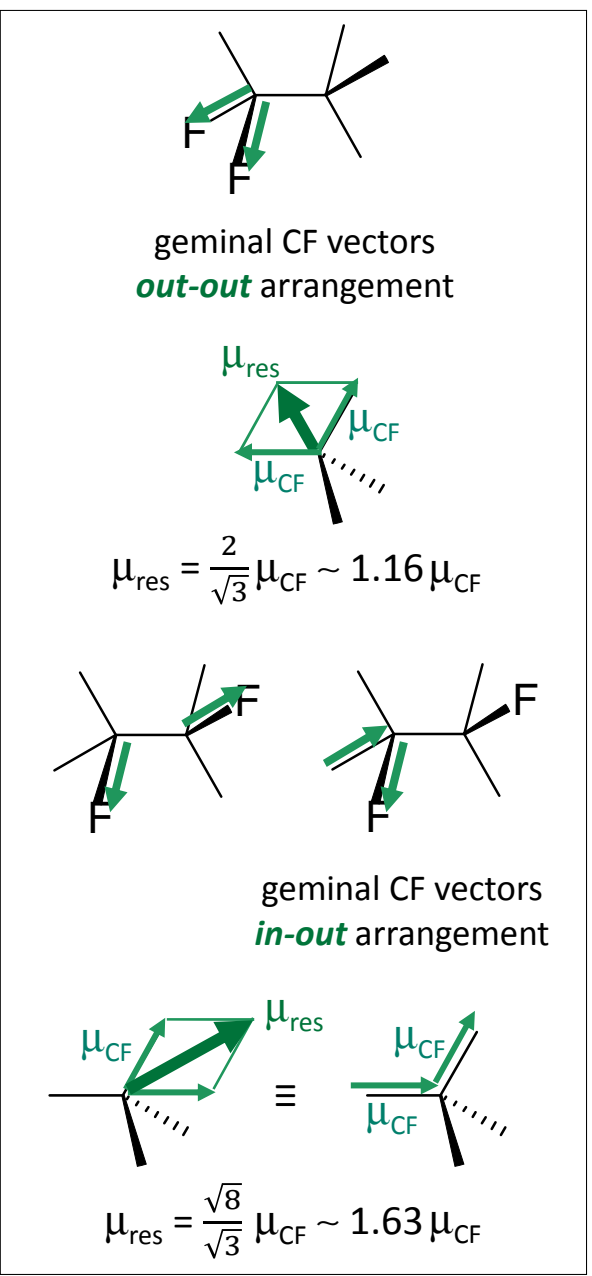

Fig. 6. Simple C-F bond vector models to rationalize the increased polarity observed and theoretically calculated for gem-difluoro and 1,2-difluoroalkane derivatives assuming perfectly staggered tetrahedral geometries for the difluoroalkanes. 
but characteristically, in the relative orientation of the two C-F bond vectors: in the geminal case, both vectors emanate outwards from their common origin; for the vicinal synclinal orientation, one vector is reversed pointing inwards towards the origin of the other vector. Since the two vectors span a rhombus, the resultant vectors for the geminal and vicinal cases correspond to the small and large diagonal, respectively. For exact tetrahedral and perfectly staggered conformations, the resultant vector is $2 / \sqrt{ } 3 \cdot \mu_{\mathrm{CF}}$ for the 'out-out' arrangement of the two $\mathrm{C}-\mathrm{F}$ bond vectors $\left(\mu_{\mathrm{CF}}\right)$, whereas it is $2 \sqrt{ } 2 / \sqrt{ } 3 \cdot \mu_{\mathrm{CF}}$ for the inout' arrangement; hence, the resultant vector is $\sqrt{2}$ times larger for vicinal staggered arrangements compared to a geminal configuration. Obviously, this result remains qualitatively correct even if exact tetrahedral configurations or staggered conformations are somewhat relaxed, or if $\mathrm{C}-\mathrm{F}$ bond polarities would differ somewhat depending on their structural contexts. Since the volume of a difluoroalkane may be assumed to be essentially independent of the topology of the fluorination pattern, the increased polarity of a vic-difluorinated alkyl group should exhibit significantly reduced lipophilicity compared to its gemdifluorinated analog. Based on the simple parameterization derived from the case of $n$-propylbenzene derivatives (see Fig. 5) a reduction of $\log P$ by almost half a $\log$ unit $(\Delta \log P \sim-0.4)$ may be estimated in going from a geminal difluoroalkyl derivative to its vicinal difluoro analog.

Of interest are then the all-gauche arrangements of three fluorine substituents in terminal bis-vicinal trifluoroalkyl derivatives, such as the 2,3,4-trifluorobutyl case (Fig. 7). Focusing on the threo configuration, assuming an all-trans CCCCbackbone with the two inner fluorines in a gauche arrangement, and applying again the simple $\mathrm{C}-\mathrm{F}$ bond vector analysis, we find that, contrary to the case of the $\mathrm{CF}_{3}$ group, where the three $\mathrm{C}-\mathrm{F}$ bonds are all geminally arranged, thus pointing in outward directions, the bis-vicinal case has one $\mathrm{C}-\mathrm{F}$ bond vector reversed, resulting in an 'in-out-out' arrangement of the three bond vectors. While the 'out-out-out' arrangement gives a resultant vector of $1 \mu_{\mathrm{CF}}$, the 'in-out-out' arrangement produces a resultant vector of almost double magnitude $\left(\sim 1.91 \mu_{\mathrm{CF}}\right.$, Fig. 7). Compared to the vicinal arrangement of a difluoroalkyl group, the bis-vicinal configuration of a trifluoroalkyl group is then predicted to be even more polar. However, the third fluorine adds additional volume and thus may just compensate the expected lipophilicity downshift based on the polarity gain. Based on the simple parameterization scheme of Fig. 5, the 1,2,3-trifluoro- and 1,2-difluoroalkyl groups are predicted to

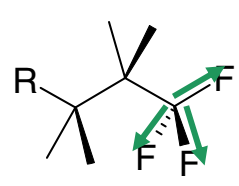

3 geminal CF vectors out-out-out arrangement

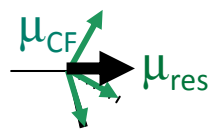

$\mu_{\text {res }}=\mu_{C F}$
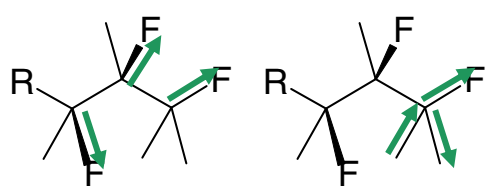

3 geminal CF vectors in-out-out arrangement

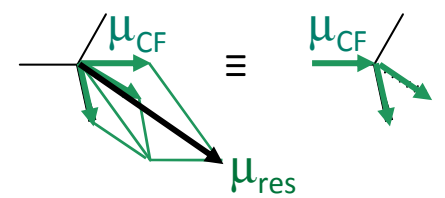

$$
\mu_{\mathrm{res}}=\frac{\sqrt{11}}{\sqrt{3}} \mu_{\mathrm{CF}} \sim 1.91 \mu_{\mathrm{CF}}
$$

Fig. 7. Bond vector analysis for terminal bisvicinal 1,2,3-trifluoro substitution pattern.

be similarly effective in reducing lipophilicity, but much more so than their geminal difluoro- or trifluoroalkyl counterparts. It is noteworthy, that any further introduction of fluorines beyond the bis-vicinal trifluoro stage would not be expected to result in an additional increase, but rather in a reduction of polarity due to unavoidable partial cancellation of $\mathrm{C}-\mathrm{F}$ bond vectors. Taking into account the concomitant volume increase upon further $\mathrm{H} / \mathrm{F}$-exchange, maximum lipophilicity lowering effects can thus be expected at the stages of vic- difluoro and (terminal) bis-vicinal trifluoro substitution patterns.

Alkoxy groups are frequently used in medicinal chemistry, with methoxy and trifluoromethoxy groups playing prominent roles. While the introduction of a methoxy group into an aromatic ring has little or no effect on the compound's lipophilicity, replacement of a methoxy group by its trifluoromethoxy counterpart results in a substantial increase in lipophilicity. Thus, benzene and anisole exhibit identical lipophilicity $\left(\log P=2.1^{[20]}\right)$, and a comparison of 24 internal neutral matched molecular pairs differing only in one methoxy group without additional substituents in the ortho, ortho'-positions reveals a nearly vanishing lipophilicity change. By contrast, replacement of an aryl-bound methoxy group by the trifluoromethoxy group produces a lipophilicity upshift by typically one $\log P$ unit as evidenced by comparison of 36 internal neutral matched molecular pairs (Fig. 8) differing by the replacement of an $\mathrm{OCH}_{3}$ by the $\mathrm{OCF}_{3}$ group only.

It is instructive to attempt a similar simplified bond polarity vector analysis for a trifluoromethoxy group as for the partially fluorinated alkyl units. By contrast to the latter, we have to account for the polar $\mathrm{C}-\mathrm{O}$ bonds of an alkoxy group. Based on electronegativity differences, ${ }^{[21]}$ the $\mathrm{C}-\mathrm{O}$ bond polarity may be expected to be reduced by $\sim 0.62$ relative to a $\mathrm{C}-\mathrm{F}$ bond. To simplify matters, we assume tetrahedral geometry around the ether oxygen atom. While this is approximately the case for aliphatic ethers, a valence angle more closely to $120^{\circ}$ is typically observed for aryl ethers due to effective anisol-type $\pi$-conjugation. Interestingly, with $\mu_{\mathrm{CO}}=0.62 \mu_{\mathrm{CF}}$ using $\mu_{\mathrm{CF}}$ $=1.85 \mathrm{D}$ as derived from methyl fluoride, dimethyl ether would be predicted to have a dipole moment of $1.32 \mathrm{D}$, in good agreement with the experimental value of 1.30 D. Applying the simplified bond vector addition scheme to the trifluoromethoxy unit and shifting the $\mathrm{C}-\mathrm{O}$ bond vectors into the origin of the three $\mathrm{C}-\mathrm{F}$ bond vectors (Fig. 9), we note that one $\mathrm{C}-\mathrm{O}$ bond vector partially compensates the three $\mathrm{C}-\mathrm{F}$ bond

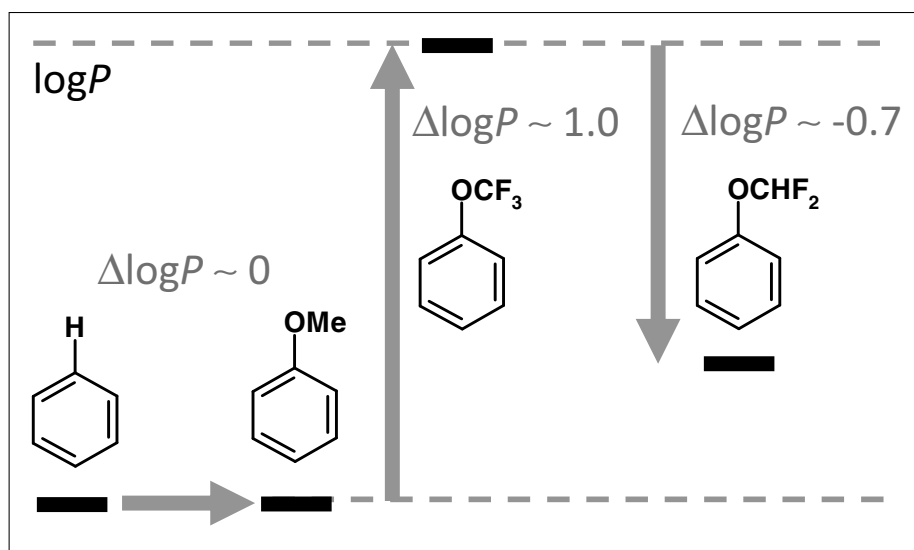

Fig. 8. Experimental $\triangle \log P$ effects for matched molecular pairs of 24 neutral aryl-H/aryl-OMe pairs $(\triangle \log P=0.05$ $\pm 0.17) ; 36$ neutral aryl-OMe/aryl-OCF pairs $(\triangle \log P=1.0 \pm$ 0.3 ); and 15 neutral aryl-OCF ${ }_{3}$ aryl-OCHF pairs $(\triangle \log P=-0.7$ $\pm 0.1)$. 


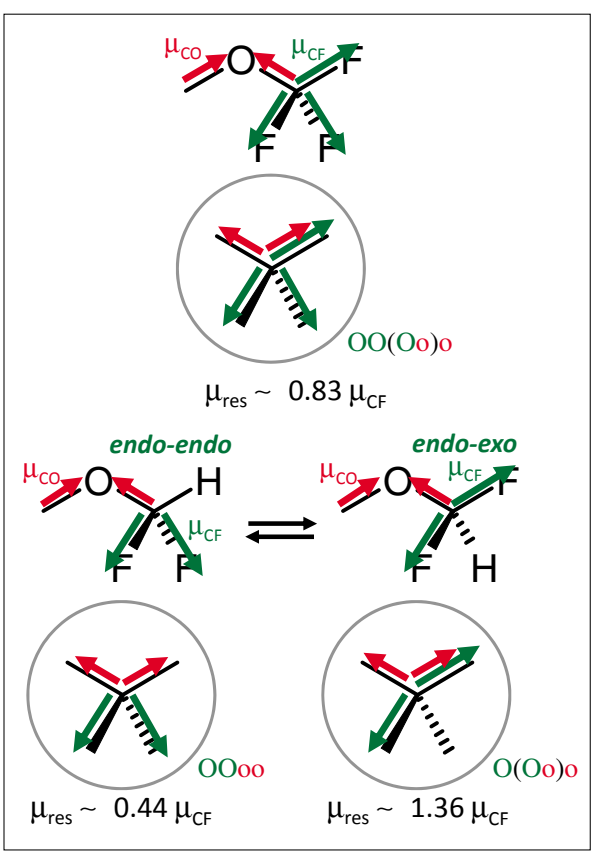

Fig. 9. Bond vector analysis for $\mathrm{C}-\mathrm{O}-\mathrm{CF}_{3}$ and $\mathrm{C}-\mathrm{O}-\mathrm{CHF}_{2}$ in the endo-endo and exo-endo conformations; $\mathrm{C}-\mathrm{O}$ bond dipole vectors $\left(\mu_{\mathrm{co}}\right.$, red) assumed to be 0.62 of $\mathrm{C}-\mathrm{F}$ bond dipole vectors $\left(\mu_{\mathrm{CP}}\right.$ green); see text. Shifting vectors to one common origin (e.g., methyl-C atom) results in characteristic vector arrangements with either three or four outgoing vectors (' $\mathrm{O}$ ' for larger C-F bond vectors, 'o' for smaller $\mathrm{C}-\mathrm{O}$ bond vectors) due to partial superposition of bond vectors. Note that four tetrahedrally outgoing vectors of equal size would cancel by symmetry.

vectors so that essentially one $\mathrm{C}-\mathrm{O}$ bond vector survives. The net result, $\mu_{\text {tot }} \sim 0.83$ $\mu_{\mathrm{CF}}$, indicates that the $\mathrm{CF}_{3} \mathrm{O}$ group is only slightly more polar than the methoxy group $\left(\mu_{\text {tot }} \sim 2 / \sqrt{ } 3 \cdot \mu_{\mathrm{CO}} \sim 0.72 \mu_{\mathrm{CF}}\right)$. Taking into account the substantial volume increase in replacing $\mathrm{CH}_{3}$ by $\mathrm{CF}_{3}$ and using the parameterization scheme derived for the fluoroalkanes (Fig. 5), we estimate a substantial $\log P$ increase of $\sim 0.8$ in going from a methoxy to a trifluoromethoxy group. While there are other factors contributing to the aryl- $\mathrm{OCH}_{3}$ /aryl- $\mathrm{CF}_{3}$ lipophilicity change, such as altered $\pi$-conjugation and partial shielding of solvation due to the conformational switch from the in-plane aryl methoxy to the orthogonal aryl trifluoromethoxy arrangement, it is nevertheless worth pointing to the intrinsic lipophilic nature of a trifluoromethoxy group.

While the trifluoromethoxy group has been around for quite some time, the difluoromethoxy group has been introduced into medicinal chemistry only more recently. ${ }^{[2]}$ The difluoromethoxy group is of particular interest as it may adopt two characteristically different conformations (Fig. 9), as evidenced by a large body of crystal structure analyses and high-level B3LYP quantum chemical calculations. ${ }^{[5 b]}$ In one conformation, both $\mathrm{C}-\mathrm{F}$ bonds are antipla- nar ('anomeric') to the O lone pair orbitals (endo-endo conformation), whereas in the other, endo-exo conformation, only one $\mathrm{C}-\mathrm{F}$ bond is 'anomeric' to an $\mathrm{O}$ lone pair orbital while the second $\mathrm{C}-\mathrm{F}$ bond is antiplanar to the $\mathrm{C}-\mathrm{O}$ bond. Simplified bond vector analyses for these two conformations (Fig. 9) indicate that there is near cancellation of bond polarities in the endoendo arrangement $\left(\mu_{\mathrm{tot}} \sim 0.44 \mu_{\mathrm{CF}}\right)$. In the endo-exo conformation one of the two outgoing $\mathrm{C}-\mathrm{F}$ bond vectors is enforced by the overlay of one $\mathrm{C}-\mathrm{O}$ bond vector, while the other $\mathrm{C}-\mathrm{O}$ bond vector constitutes a third out-going vector. Thus, in the endo-exo conformation the $\mathrm{ROCHF}_{2}$ group has three outgoing bond polarity vectors, similar to a terminal $\mathrm{CF}_{3}$ group; however, with one $\mathrm{C}-\mathrm{F}$ polarity vector significantly enhanced and one polarity vector somewhat smaller, the net result is an increased polarity of $\mu_{\text {tot }}$ $\sim 1.36 \mu_{\mathrm{CF}}$. Considering the fact that only two fluorine atoms contribute to a counterbalancing volume effect, and taking the parameterization used for the fluoroalkanes (Fig. 5), we predict the difluoromethoxy group in the endo-exo conformation to be significantly more polar $(\Delta \log \mathrm{P} \sim-0.8)$ than the trifluoromethoxy group. This contrasts the situation for the endo-endo conformation, which would be expected to be equally or even slightly more lipophilic $(\Delta \log \mathrm{P} \sim+0.1)$ than the trifluoromethoxy group. Interestingly, a Matched Molecular Pair analysis for fifteen neutral pairs of internal arylOCF 3 and arylOCHF 2 compounds revealed a substantial and highly consistent lipophilicity downshift of more than half a $\log P$ unit (Fig. 8), indicating a predominance of the polar conformation in aqueous solution. While the $\mathrm{CHF}_{2}$ unit is of particular interest due to its axially non-isotropic shape and electrostatic potential (by contrast to its isotropic $\mathrm{CH}_{3}$ or $\mathrm{CF}_{3}$ counterparts), the existence of two easily interconverting conformations for the $\mathrm{OCHF}_{2}$ group with dramatically different lipophilicity renders this group even more interesting as it can adapt to different molecular environments simply by a conformational switch.

The findings for the difluoromethoxy group stimulated similar explorations of partially fluorinated alkoxy groups, particularly difluoropropyloxy and trifluorobutyloxy groups with different fluorination patterns. While it remains to be seen to what extent the local polarity of the ether moiety combines with the polarity pattern of more distant C-F bonds, it is very likely that for more or less compact patterns of polar $\mathrm{C}-\mathrm{O}$ and $\mathrm{C}-\mathrm{F}$ bonds similar vector analyses as for (partially) fluorinated methoxy groups may provide valid guidance to promising substitution patterns. Examples are shown in Figs 10 and 11.

The cases of vic-difluoro and 1,3-diflu-

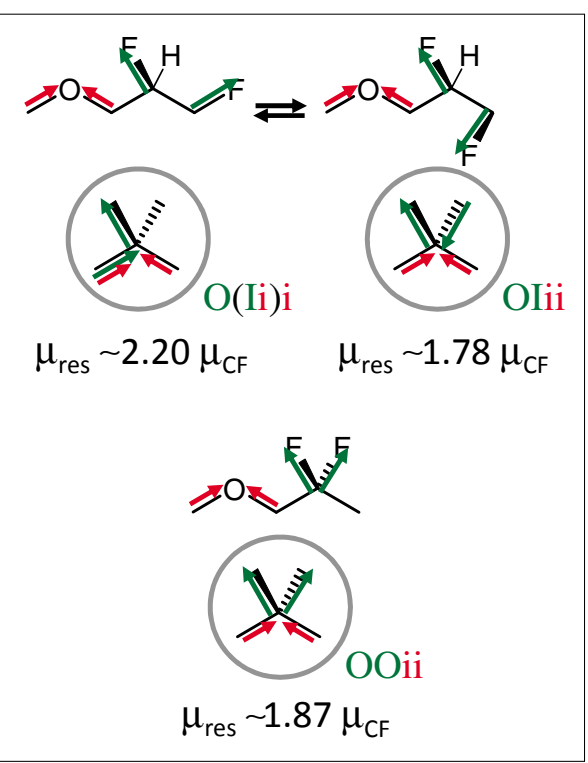

Fig. 10. Vector analyses for the 2,3-difluoropropyloxy unit (upper part) and 2,2-difluoropropyloxy group (lower part). After shifting the vectors into a common origin, the vector arrangements are designated as ' $\mathrm{O}$ ' and ' $\mathrm{l}$ ' for outward and inward pointing $\mu_{\mathrm{CF}}$ bond vectors, respectively, and ' $O$ ' and ' $i$ ' for the somewhat smaller outward and inward pointing $\mu_{\mathrm{co}}$ bond vectors, respectively; see also Fig. 9.

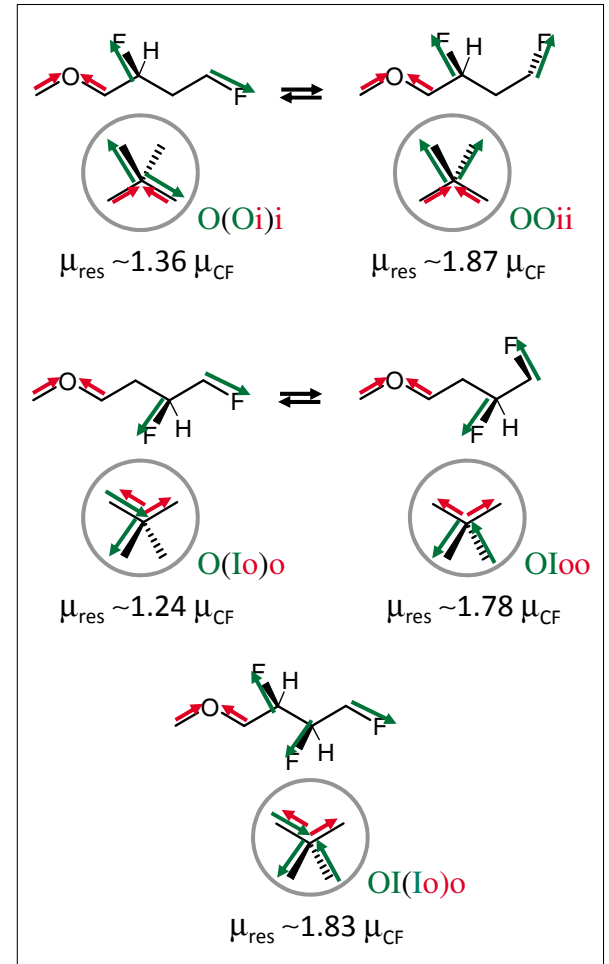

Fig. 11. Vector analyses for the 2,4-difluorobutyloxy group (top), the 3,4-difluorobutyloxy (middle), and the 2,3,4-trifluorobutyloxy groups (bottom). For signature convention of vector arrangements, see Fig. 10. As a rough guide to the outcome of the superposition of tetrahedrally arranged vectors, we note that in addition to those arrangements already mentioned for 2 or 3 vectors (see Figs 6 and 7, respectively), 4 tetrahedrally arranged vectors of equal size $(\mu)$ combine to a resultant vector of size $2 \mu$ in in-out-out-out (IOOO) or in-in-in-out (IIIO) arrangements, and to a resultant vector of size $2 \cdot 2 / \sqrt{ } 3 \cdot \mu$ $(\sim 2.3 \mu)$ for an in-in-out-out (IIOO) arrangement. 
oro substitution patterns close to an ether moiety, such as in 1-alkoxy-2,3-difluoropropane (Fig. 10, top) and 1-alkoxy-2,4difluorobutane (Fig. 11, top), respectively, are of particular interest. In both cases, the terminal fluorine may occupy either the trans-exo or the gauche-endo position with respect to the carbon backbone. For the vector analyses, we shift all $\mathrm{C}-\mathrm{F}$ and $\mathrm{C}-\mathrm{O}$ bond vectors into a common origin (e.g., $\mathrm{C}(2)$ ). Assuming all-trans carbon backbone conformations, the vector analyses for the two conformations of the 1-alkoxy-2,3-difluoropropane case then suggest a conformation with pronounced polarity when the terminal fluorine takes the transexo position, but somewhat attenuated polarity when the terminal fluorine is in the gauche-endo position. By contrast, for the 1-alkoxy-2,4-difluorobutane case, analogous bond vector analyses suggest that a polar conformation results with the terminal fluorine in the gauche-endo position, while polarity is attenuated when the terminal fluorine adopts the trans-exo position. Assuming more polar conformations to prevail in aqueous solution, the 2,3-difluoropropyloxy and the 2,4-difluorobutyloxy groups stand out as particularly interesting partially fluorinated alkoxy groups which, by contrast to their geminally difluorinated counterparts, can be expected to result in further lipophilicity reductions. Again it is of interest to note that low-energy conformations for both groups are predicted to differ markedly in polarity, thus enabling these groups easily to adapt to changing polarity of the environment.

The identification of the terminal bisvicinal trifluoro alkyl moiety as equally potent in lowering lipophilicity as the vicdifluoroalkyl unit, calls for an examination of the corresponding 2,3,4-trifluorobutyloxy group. Focusing again on the prototypic threo-1-alkoxy-2,3,4-trifluorobutane (Fig. 11, bottom) and assuming an all-trans carbon backbone with concomitant gauche arrangement of the two inner fluorine at- oms, the simple vector analysis, including the two slightly less polar $\mathrm{C}-\mathrm{O}$ bond vectors, results in a polarity comparable to, but slightly smaller than that of the terminal bis-vicinal trifluoroalkyl group (Fig. 7) due to partial compensation of $\mathrm{C}-\mathrm{F}$ and $\mathrm{C}-\mathrm{O}$ bond vectors. The situation here is reminiscent to the one encountered above when introducing more than three fluorine atoms into an alkyl group. Hence, it can be expected that the 2,3,4-trifluorobutyloxy group will be no more effective in lowering lipophilicity than either the 3,4- or the 2,4-difluorobutyloxy groups. Furthermore, any further introduction of fluorine atoms into such alkoxy groups will reduce polarity due to partial C-F bond vector cancellations so that with concomitant volume increase upon further H/F-exchange lipophilicity reduction effects will be mitigated. In summary, we may conclude that similar to the partially fluorinated alkyl cases, the vic-difluoro- and terminal bis-victrifluoroalkoxy groups stand out as most promising lipophilicity-lowering units. In addition, and by contrast to the alkyl case, the 1,3-difluoro motif is particularly promising when juxtaposed to an ether moiety, such as in the 2,4-difluorobutyloxy group.

The exchange of hydrogen by fluorine at aryl groups or the introduction of $\mathrm{CF}_{3}$ units have dominated medicinal chemistry for decades. The use of partially fluorinated alkyl and alkoxy groups has come into focus more recently. The simple bond vector analysis illustrated here for a couple of prototypic fluorination patterns for small alkyl and alkoxy groups may help to identify particularly interesting units which may significantly lower the lipophilicity of a given candidate compound. The simple analysis is also quite useful for a qualitative assessment of lipophilicity variations by conformational changes in partially fluorinated units and thus for the identification of such units as potential 'environmental adaptors'.
Received: April 1, 2014

[1] a) J. Dumas, E. Peligot, Ann. Pharm. 1835, 15, 246; b) R. Schmitt, H. Von Gehren, J. Prakt. Chem. 1870, 394; c) W. Lenz, Berichte 1877, 1135 .

[2] Thomson Reuters Integrity Database; see http:// thomsonreuters.com/integrity/

[3] a) B. E. Smart, in 'Physics and Physicochemical Properties [of fluorinated organic compounds]', Eds. M. Hudlicky, A. E. Pavlath, American Chemical Society, Monograph 187, Washington DC, 1995, p. 979; b) B. E. Smart, J. Fluorine Chem. 2001, 109, 3.

[4] a) K. Müller, ChemBioChem 2004, 5, 559; b) S. Fustero, C. del Pozo, J. Fluorine Chem. 2013, 152, 1.

[5] a) M. Molteni, C. Pesenti, M. Sani, A. Volonterio, M. Zanda, J. Fluorine Chem. 2004, 125, 1735; b) K. Müller, Ch. Faeh, F. Diederich, Science 2007, 317, 1881; c) W. K. Hagmann, J. Med. Chem. 2008, 51, 4359; d) D. O'Hagan, Chem. Soc. Rev., 2008, 37, 308.

[6] M. Morgentaler, E. Schweizer, A. HoffmannRöder, F. Benini, R. E. Martin, G. Jaeschke, B. Wagner, H. Fischer, S. Bendels, D. Zimmerli, J. Schneider, F. Diederich, M. Kansy, K. Müller, ChemMedChem 2007, 2, 1100.

[7] Q. A. Huchet, B. Kuhn, B. Wagner, H. Fischer, M. Kansy, D. Zimmerli, E. M. Carreira, K. Muller, J. Fluorine Chem. 2013, 152, 119.

[8] a) J. A. K. Howard, V. J. Hoy, D. O'Hagan, G. T. Smith, Tetrahedron 1996, 52, 12613; b) G. R. Desiraju, T. Steiner, 'The Weak Hydrogen Bond in Structural Chemistry and Biology', Oxford University Press, New York, 1999; c) C. Dalvit, A Vulpetti, ChemMedChem 2012, 7, 262; d) B.Y. Lu, Z.-M. Li, Y.-Y. Zhu, X. Zhao, Z.-T. Li, Tetrahedron 2012, 68, 8857; e) H.-J. Schneider, Chem. Sci. 2012, 3,1381.

[9] a) M. H. Abraham, P. P. Duce, D. V. Prior, D. G. Barratt, J. J. Morris, P. J. Taylor, J. Chem. Soc. Perkin Trans. II 1989, 1355; b) C. Ouvrard, M. Berthelot, Ch. Laurence, J. Chem. Soc. Perkin Trans. II 1999, 1357.

[10] a) J. D. Dunitz, R. Taylor, Chem. Eur. J. 1997, 3, 89; b) J. D. Dunitz, ChemBioChem 2004, 5, 614.

[11] J. C. Biffinger, H. W. Kim, S. G. DiMagno, ChemBioChem 2004, 5, 622.

[12] S.W. Cowan-Jacob, G. Fendrich, G. Rummel, A. Strauss, Nature 2010, 463, 501.

[13] W. Choong, Cryst. Structure Comm. 1979, 9, 27 (CSD-code: ADFGLP).

[14] J. Graton, Z. Wang, A.-M. Brossard, D. G. Monteiro, J.-Y. Le Questel, B. Linclau, Angew. Chem. Int. Ed. 2012, 51, 6176.

[15] a) C. R. S. Briggs, D. O'Hagan, J. A. K. Howard, D. S. Yufit, J. Fluorine Chem. 2003, 119,9 ; b) M. Schuler, D. O'Hagan, A. M. Z. Slawin, Chem. Commun. 2005, 4324.

[16] R. Paulini, K. Müller, F. Diederich, Angew. Chem. Int. Ed. 2005, 44, 1788.

[17] Y. Carcenac, P. Diter, C. Wakselman, M. Tordeux, New J. Chem. 2006, 30, 442.

[18] F. Leroux, ChemBioChem 2004, 5, 644.

[19] K. Kato, H. Morikawa, H. Kimoto, K. L. Kirk, Anal. Sci. 1994, 10, 693.

[20] MedChem Database, Version 2010, Daylight Chemical Information Systems, Inc.; see http:// www.daylight.com/products/medchem.html.

[21] L. Pauling, 'The Nature of the Chemical Bond', Cornell University, USA, $3^{\text {rd }}$ ed., 1960. 